\title{
(2) OPEN ACCESS \\ Clinical application of fetal genome-wide sequencing during pregnancy: position statement of the Canadian College of Medical Geneticists
}

\author{
Joanna Lazier (1) , ${ }^{1}$ Taila Hartley, ${ }^{2}$ Jo-Ann Brock, ${ }^{3}$ Oana Caluseriu, ${ }^{4}$ David Chitayat, ${ }^{5,6}$ \\ Anne-Marie Laberge, ${ }^{7}$ Sylvie Langlois, ${ }^{8}$ Julie Lauzon, ${ }^{9,10}$ Tanya N Nelson, ${ }^{11,12}$ \\ Jillian Parboosingh, ${ }^{9,10}$ Dimitri J Stavropoulos, ${ }^{13}$ Kym Boycott, ${ }^{1,2}$ \\ Christine M Armour 주, 1,14 On behalf of the Canadian College of Medical Geneticists
}

For numbered affiliations see end of article.

\section{Correspondence to}

Dr Joanna Lazier, Department of Genetics, Children's Hospital of Eastern Ontario, Ottawa, Ontario, Canada;

jlazier@cheo.on.ca

$\mathrm{KB}$ and CMA contributed equally.

$\mathrm{KB}$ and $\mathrm{CMA}$ are joint senior authors.

Received 3 April 2021 Accepted 16 July 2021
Check for updates

(C) Author(s) (or their employer(s)) 2021. Re-use permitted under CC BY-NC. No commercial re-use. See rights and permissions. Published by BMJ.

To cite: Lazier J, Hartley T, Brock J-A, et al. J Med Genet Epub ahead of print: [please include Day Month Year]. doi:10.1136/

jmedgenet-2021-107897

\section{ABSTRACT}

Purpose and scope The aim of this position statement is to provide recommendations for Canadian healthcare professionals regarding the use of genomewide sequencing (GWS) in the context of diagnostic testing of the fetus during pregnancy. This statement was developed to facilitate clinical translation of GWS as a prenatal diagnostic test and the development of best practices in Canada, but the applicability of this document is broader and aims to help professionals in other healthcare systems.

\section{Methods of statement development $A$}

multidisciplinary group was assembled to review existing literature on fetal GWS for genetic diagnosis in the context of suspected monogenic diseases and to make recommendations relevant to the Canadian context. The statement was circulated for comments to the Canadian College of Medical Geneticists (CCMG) membership-atlarge and, following incorporation of feedback, approved by the CCMG Board of Directors on 19 February 2021. Results and conclusions The use of prenatal GWS is indicated for the investigation of multiple fetal anomalies. Its use in the context of isolated fetal anomaly should be guided by available resources and current evidence, which is continually changing. During pregnancy, GWS should be ordered by, or in collaboration with, a medical geneticist. It should be used following detailed phenotyping to interrogate known disease genes, preferably using a trio approach, following detailed fetal phenotyping. Testing should be done with an overall aim to help in the management of the pregnancy, delivery and postnatal care. It should be guided by personal utility of the test for the pregnant person and clinical utility for pregnancy and birth management, as outlined herein. Genetic counselling is crucial in making the parental decision an informed decision. Chromosomal microarray analysis should be completed in parallel or prior to GWS and should be preceded by Quantitative Fluorescent PCR (QF-PCR) for detection of common aneuploidies. In normal circumstances, only pathogenic and likely pathogenic variants with a high likelihood of being associated with the identified fetal anomalies should be reported. Reporting of secondary findings, defined as purposeful analysis of variants in a set of medically actionable genes, should not, by default, be performed in the prenatal context. Laboratories should only report incidental findings that reveal risk of a significant Mendelian condition during infancy and childhood.
Should a laboratory have a policy for reporting incidental findings in medically actionable adult-onset conditions, they should only be reported with explicit opt-in consent signed by the tested individuals. Genetic counselling is crucial in disclosing the test results and the implications the results may have for the fetus. It should be emphasised that negative results do not rule out a genetic diagnosis nor guarantee a good prognosis. Postnatal phenotyping and reanalysis of existing data should be considered. Families should be given the opportunity to participate in research studies as appropriate. These recommendations will be routinely re-evaluated as knowledge of the diagnostic and clinical utility of fetal GWS during pregnancy improves.

\section{INTRODUCTION}

Approximately 2\%-4\% of pregnancies present with major fetal anomalies, which can be isolated or occur with other anomalies and can range in severity from mild to severe. ${ }^{1}$ Fetal ultrasound is the standard-of-care for identification of structural congenital anomalies and, in Canada, is typically conducted at 18-22 weeks gestation, although some centres may offer earlier scans. ${ }^{2}$ The characterisation of anomalies, and providing the option of appropriate diagnostic investigations, is a mainstay of prenatal genetics, with the purpose of understanding the aetiology and guiding pregnancy and neonatal management.

For over 30 years, the standard diagnostic investigations for fetal anomalies have included conventional prenatal cytogenetic analysis via karyotyping. Rapid aneuploidy detection for the identification of specific common fetal aneuploidies (trisomy 13, 18 and 21, sex chromosome aneuploidy and triploidy) has replaced karyotyping in many centres in Canada and, when normal, is followed by chromosomal microarray analysis to improve resolution and detect both microscopic and submicroscopic pathogenic CNVs. ${ }^{3}$ Chromosomal microarray analysis accounts for an increased diagnostic yield dependent on the anomalies, and ranges from approximately 3\%-6\% in fetuses with an isolated anomaly to greater than $10 \%$ when multiple anomalies or other fetal findings are present (eg, intrauterine growth restriction, oligohydramnios/anhydramnios). ${ }^{45}$

These diagnostic investigations can identify a number of genetic variations including aneuploidy, 
$\mathrm{CNV}$ (with specific size guidelines for reporting) and areas of loss of heterozygosity, but are unable to detect pathogenic single nucleotide variants and small insertions/deletions, which are major causes of monogenic diseases. Historically, testing for these has been done via single-gene testing; however, as the understanding of genetic heterogeneity for different disorders has increased and technical methods have improved, prenatal testing has expanded to include next-generation sequencing (NGS) methodologies such as multigene panels. Access to and use of multigene panels prenatally vary by province. These panels may have limitations; for example, they can be difficult to update and therefore slow to incorporate newly discovered genes or genes associated with ultra-rare conditions, or may not be well targeted to emerging fetal phenotypes. Moreover, fetal findings may warrant testing using more than one panel, which can be prohibitively expensive and time-consuming if pursued sequentially. Given these limitations, there is interest in pursuing less targeted genome-wide sequencing (GWS) approaches in the prenatal context.

Postnatally, GWS is widely applied for the diagnostic assessment of suspected monogenic diseases. Over 9000 patients have now been reported in the scientific literature. A recent metaanalysis found a pooled diagnostic yield of $41 \%$ for whole genome sequencing and $36 \%$ for exome sequencing (ES), ${ }^{6}$ and hundreds of thousands of additional patients have now been sequenced in clinical diagnostic laboratories. Given its success in the postnatal context, it is reasonable to presume this test would result in an increased diagnostic yield in the prenatal context. Studies are emerging showing diagnostic utility for fetal ES in the context of highly select prenatal indications, and, as a result, clinical ES for fetal anomalies is increasingly being sought. Determining the optimal diagnostic testing options for a fetus with anomalies and the scenarios under which the testing should be considered has therefore become important.

At the time of writing, fetal GWS is not widely available in Canada nor funded by all provinces, currently limiting its use. This document addresses the clinical use of GWS for fetuses during pregnancy in Canada and aims to provide anticipatory guidance for the implementation of fetal GWS as it becomes available in each province. While the document was developed to guide practice in Canada, the applicability of this statement is not confined to Canadian borders and may be of interest to clinicians and health authorities internationally.

The Canadian College of Medical Geneticists (CCMG) is a national professional organisation that ensures adherence to high standards in the delivery of medical genetics services in Canada, including standards for the training, competence and behaviour of those delivering these services. This position statement provides recommendations for fetal GWS during pregnancy for those providing prenatal genetic services in Canada. The statement was developed by a multidisciplinary working group representing the CCMG clinical and laboratory disciplines as well as the practices of maternal fetal medicine, genetic counselling and bioethics. The group met by teleconference on several occasions to draft this position statement. It was circulated to the CCMG membership on 7 December 2020 for comment by the membership and approved by the CCMG Board of Directors on 19 February 2021.

\section{DEFINITIONS FOR THIS DOCUMENT}

- Clinical utility: the ability of a test result to lead to an improved health outcome that will benefit a patient.

- Exome sequencing: a process used to determine the DNA sequence of most of the protein-encoding exons found in the genome of an individual.
- Genetic heterogeneity: the occurrence of similar or identical phenotypes caused by disruption of different genes.

- Genome sequencing: a process used to determine the sequence of most of the DNA content encompassing the entire genome of an individual.

- Genome-wide sequencing: a generic term for the process used to determine the sequence of most, if not all, genes and their associated analysis, including variant interpretation whether by large virtual gene panel, exome or genome.

- Incidental finding: genetic variant that is associated with a disorder unrelated to the primary indication for testing and is identified by chance during analysis.

- Medically actionable result: a result that can impact a patient's clinical management because treatment or preventive measures are available.

- Monogenic (Mendelian) condition: a genetic condition resulting from altered function of a single gene/locus.

- Multifactorial inheritance: non-monogenic inheritance of specific phenotypes that are determined by the interaction of multiple genetic and environmental factors.

- Multigene panel sequencing: targeted sequencing, primarily by NGS, of a selection of genes associated with a specific clinical indication.

- Next-generation sequencing: massively parallel sequencing techniques that allow for rapid assessment of multiple genes to entire genomes. These methods can be used to sequence gene panels as well as exomes or genomes.

- Personal utility: the ability of a test result to be used for decisions, actions or understanding relevant to the patient beyond healthcare-related management, including facilitating future planning and facilitating reproductive planning.

- Primary finding: genetic variant that is related to the primary indication for testing.

- Primary indication: the constellation of clinical features that prompt diagnostic evaluation by GWS.

- Secondary finding: genetic variant identified that is associated with a disorder unrelated to the primary indication for testing but is deliberately searched for by the clinical laboratory. For example, this could include pathogenic or likely pathogenic variants in genes defined by the American College of Medical Genetics and Genomics (ACMG) as causing 'actionable and highly penetrant' medical conditions. ${ }^{7}$

\section{CONSIDERATIONS}

\section{Diagnostic yield of fetal GWS during pregnancy}

In comparison with the postnatal literature, there is a relative paucity of scientific literature regarding the application of GWS technologies in pregnancy. A recent scoping review found that the diagnostic yield varied dramatically between studies. ${ }^{8}$ This was attributed to significant differences in study design, particularly criteria for inclusion (eg, specific fetal findings or anomalies), timing of testing and methods for analysis. ${ }^{8}$ Most studies are retrospective case series of highly selected and small numbers (typically $<50$ ) of fetuses with diverse indications for testing (eg, stillbirths, fetuses from terminated pregnancies and other highly selected cases based on anomalies present), which make them difficult to compare and generalise with regard to the diagnostic utility of this testing.

Two large prospective studies using a trio ES approach have been published that recruited singleton pregnancies with structural anomalies, including increased nuchal translucency, identified on prenatal ultrasound. ${ }^{9}{ }^{10}$ In Lord et al, ${ }^{9}$ genetic diagnoses were identified in $8.5 \%$ (52 of 610 ) of fetuses, and in Petrovski et $a l^{10}$ genetic diagnoses were identified in 10\% (24 of 234). 
Both studies showed that ES for isolated anomalies has a lower yield compared with those with multisystem anomalies, with approximately $6 \%$ yield for isolated anomalies and $15 \%$ and $19 \%$ yields for multisystem anomalies.

When considering specific isolated anomalies, ascertaining detailed information on diagnostic yields is challenging. When reported, the results are based on a small number of fetuses in each single anomaly category and it is not possible to combine the data across different studies given differences in study design. The two large prospective studies mentioned above ${ }^{910}$ reported higher diagnostic yields for some anomalies; however, there was variation even between these two studies. Evidence focused on specific indications is continuing to emerge. In a recent relatively large study, Sparks et $a l^{11}$ identified a genetic aetiology in $29 \%$ of fetuses with nonimmune fetal hydrops (NIFH), revealing a high diagnostic rate for this particular presentation. Ultimately these papers provide initial work that alludes to the potential utility of ES for certain isolated anomalies. Thus, despite there being limited evidence at the time of this statement to support the use of GWS for any specific isolated fetal anomaly (beyond NIFH), we have delineated a set of guiding principles for the use of fetal GWS that can be applied when additional evidence becomes available.

\section{Balancing diagnostic yield with personal and clinical utility}

In addition to assessing the likelihood of finding a diagnosis using GWS (eg, the diagnostic yield for a given indication), clinicians must also consider the test in the context of its potential personal and clinical utility for their patient. Personal utility in genetics has been used to refer to the utility beyond health management, such as allowing the patient to prepare psychologically for the future and facilitating reproductive planning. ${ }^{12}$ In contrast, clinical utility is defined as 'leading to an improved health outcome, including diagnosis, treatment, management, or disease prevention, that will benefit a patient or his/her family members'. ${ }^{13}$ In the prenatal context, this includes options for ongoing fetal and maternal surveillance, implications for the delivery, and postnatal management of the neonate. The application of usual definitions of utility is complicated in the prenatal context by limited treatment options during pregnancy. However, providing a diagnosis helps patients make informed decisions regarding the option of pregnancy interruption. Utility in each case is also influenced by the gestational age and timing of results.

Thus far, there is very little evidence regarding personal and clinical utility following prenatal GWS. Regardless, the Working Group strongly believes that both of these concepts should be considered while making national recommendations. We are of the opinion that it will be a balance between the diagnostic yield and personal and clinical utility (ie, the usefulness of a diagnosis in informing prognosis and guiding management for the specific patient and care team) that will be the most useful approach for determining appropriate application of GWS as a diagnostic test during pregnancy. Consider the scenario in which fetal imaging reveals multiple severe fetal anomalies_in such a scenario, despite a higher expected diagnostic yield, GWS may actually have less additional prognostic value, because in cases of severe anomalies the fetal outcome can often be predicted to be poor without additional information. Patients may thus be able to make decisions (eg, interruption of pregnancy or plans for postnatal management) independent of a genetic diagnosis. In such cases, there may be harm related to the anxiety from postponing decision-making until the GWS results are available, as well as the uncertainty based on unclear results, the risk of false reassurance with a negative result and the possibility of identifying incidental findings. In contrast, identification of a genetic diagnosis in a fetus with an apparently isolated fetal anomaly, although with a lower diagnostic yield, has the potential to significantly inform the prognosis and thus could significantly impact the management of that pregnancy.

Pretest counselling using a patient-centred approach can help patients understand what to expect and decide if testing, when clinically appropriate, would have utility for them. In any deferred testing scenario, postnatal GWS should remain available after interruption of pregnancy or at birth when more phenotypic information is available.

\section{Challenges in the application of clinical GWS during pregnancy}

There are a number of challenges associated with the use of GWS in the prenatal context that should be considered, including complexities associated with clinical phenotyping, technical considerations, timing considerations and interpretation of the results.

Perhaps the most significant challenge is that of adequate and accurate phenotyping. Deep phenotyping is limited to prenatal structural findings, which can be limited due to gestational age, general imaging limitations and the fact that key diagnostic features may not be a structural finding. In addition, some phenotypic features of a disorder may not yet have developed, and what has developed may appear different from our common, postnatally biased, understanding of the condition. The full prenatal phenotypic spectrum of many diseases is not yet known.

Technical aspects and issues with timeline introduce further complexities, as with all prenatal genetic testing. Invasive prenatal procedures (eg, chorionic villus sampling or amniocentesis) are needed to obtain fetal DNA samples for testing. Cell culture may be required, which adds to the turnaround time (TAT) of the test. Precautions must be taken to ensure the DNA tested reflects the affected fetal DNA, including maternal cell contamination studies and testing in multifetal pregnancies. Furthermore, in many cases, single-gene tests or multigene panels may still be more appropriate than GWS. Advantages to more targeted testing include factors such as depth of coverage, decreased need for a trio approach, genespecific reporting expertise, decreased chance of incidental findings and potentially faster TAT, among others.

Timing considerations such as its integration with other genetic tests and the TAT of these tests need to be considered. Once the decision is made to pursue fetal GWS, 'rapid' or 'expedited' results are often sought to guide pregnancy management and prolonged TATs for GWS may limit available options. ${ }^{14}$ At the current time, clinical GWS testing is available in some laboratories with a rapid TAT, but is usually more costly both in terms of financial and laboratory resources than standard TATs. This is an important consideration in the context of a single payer system like Canada's, particularly given the limited evidence regarding the personal and clinical utility of the test.

GWS introduces the potential to report a complex variety of results including pathogenic, likely pathogenic and variants of uncertain significance (VUS) possibly related to the primary indication for testing, as well as secondary and incidental findings. Some of these findings may be difficult to interpret in the context of an ongoing pregnancy and may further exacerbate an already stressful, pressured and time-sensitive situation. For example, VUS are harder to investigate in a prenatal context than postnatally, as secondary sources of information may not be available. In addition, VUS do not provide sufficient information on which to base clinical decisions and may add anxiety 
and confusion, with little benefit to the patient. At the time of writing this position statement, there is little, if any, evidence regarding the benefits and harms of identifying secondary and incidental findings in a fetus.

\section{The Canadian context}

Canada's publicly funded healthcare system is guided by the Canada Health Act and provides universal coverage for medically necessary healthcare services, although each provincial and territorial healthcare system decides how best to define specific funding parameters for their jurisdiction. In addition, physicians practising within each province must balance their obligations to the individual patient with the utilisation of finite resources when considering diagnostic testing options. Interruption of pregnancy is included in these services with no legal definition regarding gestational age limitations, although there are significant and variable non-legal barriers to access between provinces. ${ }^{14}$ The recommendations herein provide a basis for standard practice for the use of clinical GWS during pregnancy in Canada.

\section{RECOMMENDATIONS FOR THE USE OF CLINICAL GWS IN PREGNANCY \\ Pretest considerations \\ General considerations}

- Clinical GWS should only be used to interrogate the genome for sequence variants in genes known to cause disease.

- Given the complexity of interpretation and counselling, clinical GWS should only be ordered in pregnancy by, or in collaboration with, a medical geneticist with expertise in prenatal diagnosis and care, the use of the technology, and clinical interpretation of the results.

- Phenotyping is key in deciding if GWS is indicated and is critical to the interpretation of the data. As such, clinicians should ensure that reliable and detailed clinical information is collected and communicated to assist in the interpretation of results both in the laboratory and clinical settings. This includes detailed imaging of the fetus by centres experienced in detection of fetal anomalies and potentially other investigations (eg, ultrasound, fetal echo, fetal MRI, biochemical tests). Additional important information includes prior test results as well as a three-generation family history, including any information about consanguinity.

- Whenever possible, GWS trios (testing of fetus-motherfather) should be performed. Having access to the trio helps interpret variants by increasing sensitivity for detecting de novo and compound heterozygous variants; this facilitates an increased diagnostic yield based on the ACMG variant classification criteria. ${ }^{15}$ In rare circumstances when either biological parent sample is not available (eg, sperm and/or egg donor), the fetal indications should be correspondingly robust to ensure an adequate detection rate (eg, multiple congenital anomalies).

\section{Fetal indications}

- Clinical GWS could be considered in fetuses with an abnormal phenotype suggesting a monogenic aetiology, but for which a specific diagnosis is not suspected. Clinical GWS should not be performed in a fetus with a normal phenotype.

- Currently, evidence supports the use of clinical GWS in the diagnostic investigation of congenital anomalies affecting more than one system. Consensus opinion among the Working Group was that the following findings should be considered an anomaly: unexplained intrauterine growth retardation (growth $<3$ rd percentile), unexplained overgrowth ( $>97$ th percentile), increased nuchal translucency $(\geq 3.5 \mathrm{~mm})$, and unexplained polyhydramnios and oligohydramnios.

- Clinical GWS may be considered in cases of apparently isolated structural fetal anomalies, although at present evidence is generally limited as to diagnostic yield and is dependent on the specific anomaly. As discussed above, there is additional evidence for the case of hydrops, which is a single presentation with many underlying causes; this supports the use of GWS in such cases. Decisions regarding clinical GWS in the context of isolated anomalies and other presentations will need to be made at the jurisdictional level based on available resources and evolving evidence on diagnostic and clinical utility.

- The following fetal findings should not be considered eligible anomalies for GWS: isolated neural tube defect (other than encephalocoele), gastroschisis, amniotic bands or soft markers.

- Clinical GWS should not be used when maternal diseases or exposures to teratogens are suspected to be the cause of the fetal abnormalities.

\section{Integration into the prenatal diagnostic care pathway}

- Rapid aneuploidy diagnosis must be completed prior to GWS. Chromosomal microarray should be completed in parallel, or prior to, GWS, depending on the urgency of test results.

- Clinicians should consider whether single-gene testing or comprehensive multigene panels are a better approach given that they cost less (although this may change over time), may take less time and usually guarantee better coverage.

Many panel-based tests report VUS in the prenatal setting, which may be desired in specific cases. This approach may be appropriate for fetuses in which the anomalies are associated with low to moderate genetic heterogeneity and those in which a recognisable syndrome or genetic condition is suspected, such as clinical features suggestive of Noonan syndrome.

\section{Personal and clinical utility}

- A patient-centred approach is of paramount importance. The information sought by the patient and the timing required for the availability of this information are key to the personal utility of this testing during pregnancy and its timing in the diagnostic care pathway.

- Timing of testing and the decision of whether to use 'rapid' GWS should be directed by the clinical presentation (see Fetal indications section) and the potential impact on pregnancy management. Given the increased cost, rapid testing should be reserved for situations in which the standard TAT is insufficient for pregnancy management, while standard TATs are appropriate in other clinical scenarios. For example:

- If the pregnant person will base a decision regarding continuation or interruption of pregnancy on the result, then rapid prenatal GWS may be appropriate.

- If the fetal malformations increase the likelihood of a complicated delivery or neonatal course, and understanding the cause and natural history of the condition could guide the degree of intervention, then prenatal GWS may be appropriate but less urgency may be required. 


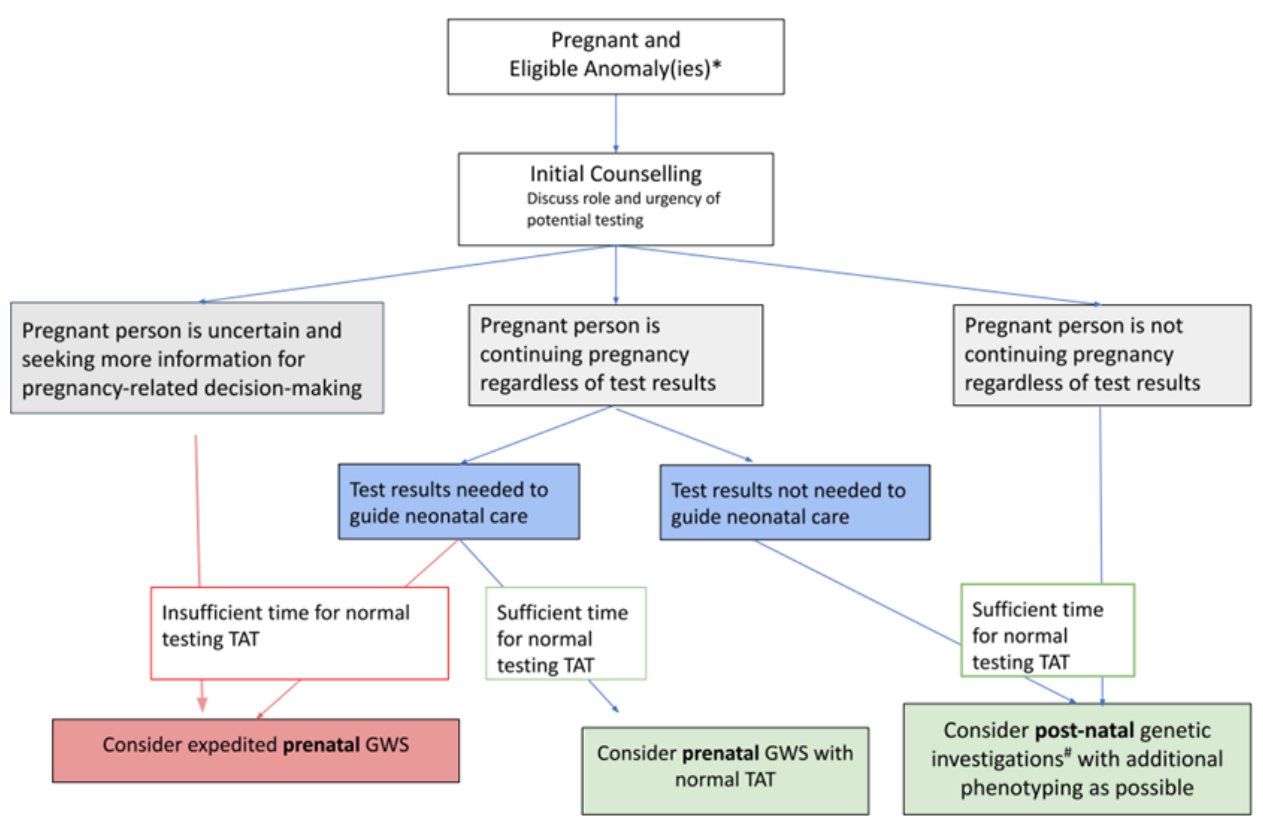

Figure 1 Decision aid to determine the speed and timing of GWS to be ordered in the diagnostic assessment of an affected pregnancy. Other relevant tests (eg, rapid aneuploidy detection and microarray) should be integrated as appropriate. This suggested framework is based on our current understanding and will be re-evaluated over time as new data become available. CCMG, Canadian College of Medical Geneticists; GWS, genome-wide sequencing; TAT, turnaround time. *See indications section of recommendations; \#See CCMG exome guidelines.

- If the decision to continue or interrupt the pregnancy has already been made by the pregnant person and the results of this testing are not anticipated to have an impact on the perinatal management, it is recommended that the testing be done postnatally (either on the neonate or the fetus) following detailed postnatal phenotyping figure 1.

- An approach to decision making is shown in figure 1.

\section{Genetic counselling}

- It is imperative that genetic counselling be undertaken prior to testing by a qualified professional with a thorough understanding of the complexity of clinical GWS and experience in the prenatal setting, with documentation of the discussion in the medical records.

- Each pregnant person's unique background can lead to variable understanding and assumptions about the predictive value of genetic testing results. Unbiased and thorough counselling of patients, including the use of a medical interpreter as needed, should occur to ensure a full understanding of the information provided.

- Counselling should address the anomaly or anomalies identified by fetal imaging and discuss the known information regarding the differential diagnosis and potential outcomes (eg, expected need for surgery after birth in case of heart defect, etc).

- Counselling should address, in an unbiased fashion, the pregnant person's options based on the available information. It should address that genetic testing may or may not be able to provide further information for decision-making and that, if results are identified, they may indicate a range of outcomes.

- Counselling should emphasise that a negative GWS report does not rule out a genetic condition nor guarantee absence of additional findings or issues (eg, cognitive impairment, deafness, blindness, etc).
Counselling should also include the following:

- An explanation of the testing and discussion of how the results may be used during the pregnancy.

- Information regarding the limitations of the test methodology (eg, CNV detection ability, trinucleotide repeats, imprinting, etc).

- Discussion of expected results and what will and will not be reported from the test, including variant classes (pathogenic and likely pathogenic only), primary findings and various kinds of incidental findings, and the choices pertaining thereto.

- The importance of phenotyping in the interpretation of GWS results and implications related to the use of the test prenatally and postnatally.

- Need for parental samples and information about what will and will not be reported with respect to variants identified in the parental samples, including the identification of inherited pathogenic or likely pathogenic variants.

- An explanation of what will happen with the phenotypic and genotypic data, including how long it will be stored and if and when additional analysis or reanalysis could be performed in the future.

- Potential issues related to insurance and discrimination.

- The ability of the test to detect non-biological relationships and how it would be disclosed.

- Written documentation of informed consent, obtained prior to testing.

\section{REPORTING RECOMMENDATIONS}

\section{Clinical testing and results reporting}

- Testing should be performed in a laboratory with appropriate clinical accreditation for provision of clinical GWS. The laboratory should have expertise in fetal genetic testing, and an understanding of, and analysis approach 
that addresses, the limitations/risks related to sample source.

- It is recommended that parental samples be sequenced and analysed simultaneously with the fetal sample to facilitate the interpretation of the fetal findings, but should not be reported individually.

- Laboratories should be explicit in what results they will report. As a general rule, only pathogenic and likely pathogenic variants associated with the identified anomalies should be reported. However, there are very rare exceptional circumstances where a VUS may be reported, for example a VUS with very strong evidence of pathogenicity ${ }^{15}$ or a VUS in trans with a pathogenic or likely pathogenic variant in a gene known to cause a recessive disease. Benign/ likely benign variants should not be reported.

- Laboratory reports should meet clinical recommendations for interpretation and reporting of NGS results, with consideration to the limitations of prenatal phenotype information. $^{16}$

- The laboratory report should include an interpretation by an appropriately clinically certified $\mathrm{PhD}$ or MD laboratory geneticist; in Canada, this would typically be an individual with CCMG or equivalent certification. ${ }^{16} 17$

- The laboratory does not have the obligation to later recontact patients to advise of updated findings not included in the original prenatal GWS report, but should have clear policies as to the process by which data may be reanalysed.

\section{Reporting of incidental and secondary findings}

Prenatal GWS is employed with the intent of correlating clinical findings with potential underlying monogenic aetiologies and to inform management of the current pregnancy or neonatal care. Due to the nature of GWS, there is potential for the identification of variants with implications that are unrelated to the primary indication for testing. Pathogenic or likely pathogenic variants unrelated to the primary indication for testing can be identified by chance (incidentally; incidental findings) or purposely using a list of actionable genes with the intent to report variants unrelated to the primary indication (secondary findings). Trio analysis is such that the variants analysed will only be those detected in the fetus; thus, incidental findings will only be detected in the parents when first detected in the fetus, unless otherwise actively sought as secondary findings in the parental samples.

There is currently no published information regarding the reporting of incidental or secondary findings in pregnancy, including the benefits and harms to families. We therefore do not endorse the automatic/default reporting of secondary findings for the fetus and the committee recommends that laboratories/ jurisdictions should have clear policies for the reporting of incidental findings that are shared with care providers and families.

- Laboratories should not purposefully analyse prenatal GWS data for diseases unrelated to the primary reason for referral (eg, secondary findings), even if the results might be medically actionable for the fetus or the parents.

- Incidental findings unintentionally identified that show a pathogenic or likely pathogenic variant that reveals a fetal risk for a significant Mendelian paediatric-onset condition, whether or not medically actionable, should be reported.

- Incidental findings unintentionally identified that show a pathogenic or likely pathogenic variant revealing a fetal susceptibility for medically actionable adult-onset diseases should not by default be reported. Should a laboratory have a policy for reporting incidental findings in medically actionable adult-onset conditions, they should only be reported with explicit opt-in consent signed by the tested individuals.

- It is recommended that laboratories do not report fetal carrier status unless directly related to the primary indication for testing.

- Reporting of parental incidental findings should be limited to only those present in the fetus.

- Both laboratories and clinics should have clear policies on how non-biological relationships are conveyed and reported.

\section{POST-TEST RECOMMENDATIONS \\ Return of results}

- The medical geneticist should review the report and incorporate the findings with other relevant medical considerations when discussing the results with the patient; if additional information becomes available that would result in reclassification of the rare reported VUS, the updated information should be relayed to the reporting laboratory for reclassification and interpretation.

- If a causative variant is identified in the fetus:

- Standard-of-care genetic counselling and anticipatory management should be provided.

- Counselling should review that, even in well-described conditions, ambiguity about the full range of potential phenotypes remains and cannot be clarified prenatally.

- If a previously undiagnosed parent is also identified by testing, genetic counselling and appropriate management or referrals should be provided.

If no causative variant is identified in the fetus:

- Patients should be counselled that clinical GWS is not a rule-out test. Thus:

- In spite of a negative result, the anomaly(ies) may still be genetic and/or monogenic and possibly associated with a poor prognosis.

- Other disorders should be considered based on the clinical presentation as appropriate, particularly those that would not be assessed by GWS (eg, imprinting or trinucleotide repeat expansion disorders).

- Postnatal reanalysis could be requested if there is additional phenotype information to contribute to analysis or if knowledge of postnatally reportable VUS would be helpful.

- Future analysis may lead to a diagnosis at a later date when more genetic knowledge becomes available. Such reanalysis should be considered using the same laboratory guidelines as for postnatal patients.

- All patients/families should be given the opportunity, at the appropriate time, to participate in current or future research studies to understand the relationship between genetic variants and developmental anomalies. Explicit informed consent should be obtained for research studies.

\section{CONCLUSIONS}

We recognise that GWS is currently not uniformly and widely available in prenatal settings in many Canadian provinces. We recommend that Canadian jurisdictions should plan for the implementation of GWS in the prenatal setting and we propose that this position statement serve to provide direction regarding the use of this testing across Canada. The recommendations outlined herein were developed based on current evidence and will evolve further as additional evidence on diagnostic yield and clinical utility becomes available. Coordinated efforts in 
evaluative health services research should be made to retrieve the clinical outcomes of this testing to inform future health system decision-making. In addition, such real-world outcomes will improve implementation with time. Importantly, appropriate collection of such data may provide the basis to further refine the concept of personalised clinical utility. Finally, while these recommendations will be very useful in the Canadian context, they may also prove useful to other countries in the process of integrating prenatal GWS into their systems of care.

\section{Author affiliations}

'Department of Genetics, Children's Hospital of Eastern Ontario, Ottawa, Ontario, Canada

${ }^{2}$ Children's Hospital of Eastern Ontario Research Institute, University of Ottawa, Ottawa, Ontario, Canada

${ }^{3}$ Departments of Pathology \& Laboratory Medicine and Obstetrics \& Gynaecology, IWK Health Centre, Halifax, Nova Scotia, Canada

${ }^{4}$ Medical Genetics Clinic, University of Alberta, Edmonton, Alberta, Canada

${ }^{5}$ The Prenatal Diagnosis and Medical Genetics Program, Department of Obstetrics and Gynecology, University of Toronto, Toronto, Ontario, Canada

'Division of Clinical and Metabolic Genetics, Department of Pediatrics, The Hospital for Sick Children, University of Toronto, Toronto, Ontario, Canada

${ }^{7}$ Service de Génétique Médicale, CHU Sainte-Justine and Département de Pédiatrie, Université de Montréal, Montréal, Quebec, Canada

${ }^{8}$ Department of Medical Genetics, University of British Columbia, Vancouver, British Columbia, Canada

${ }^{9}$ Department of Medical Genetics, Cumming School of Medicine, University of Calgary, Calgary, Alberta, Canada

${ }^{10}$ Alberta Children's Hospital Research Institute for Child and Maternal Health, Cumming School of Medicine, University of Calgary, Calgary, Alberta, Canada

${ }^{11}$ Department of Pathology and Laboratory Medicine, BC Children's Hospital and BC Women's Hospital Vancouver, Vancouver, British Columbia, Canada

${ }^{12}$ Department of Pathology and Laboratory Medicine, The University of British Columbia, Vancouver, British Columbia, Canada

${ }^{13}$ Genome Diagnostics, The Hospital for Sick Children, University of Toronto, Toronto, Ontario, Canada

${ }^{14}$ Prenatal Screening Ontario (PSO), Better Outcomes Registry \& Network (BORN) Ontario, Ottawa, Ontario, Canada

Contributors $\mathrm{LL}, \mathrm{TH}, \mathrm{KB}$ and $\mathrm{CMA}$ were responsible for identifying the literature, summarising working group meetings and drafting the manuscript. All authors contributed to the conception and design of the position statement, interpretation of data, critical revisions contributing to intellectual content and approval of the final version of the manuscript.

Funding We gratefully acknowledge the Care4Rare Canada Consortium (Genome Canada and CIHR; OGI-0147) for support. TH was supported by a Frederick Banting \& Charles Best Canada Graduate Scholarship Doctoral Award from CIHR. KB was supported by a CIHR Foundation Grant (FDN-154279) and a Tier 1 Canada Research Chair in Rare Disease Precision Health.

Competing interests TN: spouse employed by Illumina.

Patient consent for publication Not required.

Provenance and peer review Not commissioned; externally peer reviewed.

Open access This is an open access article distributed in accordance with the Creative Commons Attribution Non Commercial (CC BY-NC 4.0) license, which permits others to distribute, remix, adapt, build upon this work non-commercially, and license their derivative works on different terms, provided the original work is properly cited, appropriate credit is given, any changes made indicated, and the use is non-commercial. See: http://creativecommons.org/licenses/by-nc/4.0/.

\section{ORCID iDs}

Joanna Lazier http://orcid.org/0000-0003-2709-5049

Christine M Armour http://orcid.org/0000-0003-1767-1911

\section{REFERENCES}

1 Persson M, Cnattingius S, Villamor E, Söderling J, Pasternak B, Stephansson O, Neovius M. Risk of major congenital malformations in relation to maternal overweight and obesity severity: cohort study of 1.2 million singletons. BMJ 2017;357:j2563.

2 Cargill Y, Morin L. No. 223-Content of a complete routine second trimester obstetrical ultrasound examination and report. J Obstet Gynaecol Can 2017:39:e144-9.
3 Armour CM, Dougan SD, Brock J-A, Chari R, Chodirker BN, DeBie I, Evans JA, Gibson WT, Kolomietz E, Nelson TN, Tihy F, Thomas MA, Stavropoulos DJ, On-Behalf-Of the Canadian College of Medical Geneticists. Practice guideline: joint CCMG-SOGC recommendations for the use of chromosomal microarray analysis for prenatal diagnosis and assessment of fetal loss in Canada. J Med Genet 2018;55:215-21.

4 Wapner RJ, Martin CL, Levy B, Ballif BC, Eng CM, Zachary JM, Savage M, Platt LD, Saltzman D, Grobman WA, Klugman S, Scholl T, Simpson JL, McCall K, Aggarwal VS, Bunke B, Nahum O, Patel A, Lamb AN, Thom EA, Beaudet AL, Ledbetter DH, Shaffer LG, Jackson L. Chromosomal microarray versus karyotyping for prenatal diagnosis. N Engl J Med 2012;367:2175-84.

5 Hillman SC, Pretlove S, Coomarasamy A, McMullan DJ, Davison EV, Maher ER, Kilby MD. Additional information from array comparative genomic hybridization technology over conventional karyotyping in prenatal diagnosis: a systematic review and metaanalysis. Ultrasound Obstet Gynecol 2011;37:6-14.

6 Clark MM, Stark Z, Farnaes L, Tan TY, White SM, Dimmock D, Kingsmore SF. Metaanalysis of the diagnostic and clinical utility of genome and exome sequencing and chromosomal microarray in children with suspected genetic diseases. NPJ Genom Med 2018;3:16.

7 Kalia SS, Adelman K, Bale SJ, Chung WK, Eng C, Evans JP, Herman GE, Hufnagel $S B$, Klein TE, Korf BR, McKelvey KD, Ormond KE, Richards CS, Vlangos CN, Watson $\mathrm{M}$, Martin $\mathrm{CL}$, Miller DT. Recommendations for reporting of secondary findings in clinical exome and genome sequencing, 2016 update (ACMG SF v2.0): a policy statement of the American College of medical genetics and genomics. Genet Med 2017:19:249-55.

8 Pratt M, Garritty C, Thuku M, Esmaeilisaraji L, Hamel C, Hartley T, Millar K, Skidmore B, Dougan S, Armour CM. Application of exome sequencing for prenatal diagnosis: a rapid scoping review. Genet Med 2020;22:0918:1925-34.

9 Lord J, McMullan DJ, Eberhardt RY, Rinck G, Hamilton SJ, Quinlan-Jones E, Prigmore E, Keelagher R, Best SK, Carey GK, Mellis R, Robart S, Berry IR, Chandler KE, Cilliers D, Cresswell L, Edwards SL, Gardiner C, Henderson A, Holden ST, Homfray T, Lester T, Lewis RA, Newbury-Ecob R, Prescott K, Quarrell OW, Ramsden SC, Roberts E, Tapon D, Tooley MJ, Vasudevan PC, Weber AP, Wellesley DG, Westwood $P$, White H, Parker M, Williams D, Jenkins L, Scott RH, Kilby MD, Chitty LS, Hurles ME, Maher ER, Bateman M, Campbell C, Campbell J, Carey G, Cohen K, Collingwood E, Constantinou P, Delmege C, Ellis R, Evans J, Everett T, Pinto CF, Forrester N, Fowler E, Hamilton S, Healey K, Hudson R, Lester T, Lewis R, Marton T, Mehta S, Park SM, Rowland J, Steer J, Taylor EJ, Wilson E, Prenatal Assessment of Genomes and Exomes Consortium. Prenatal exome sequencing analysis in fetal structural anomalies detected by ultrasonography (page): a cohort study. Lancet 2019;393:S0140-6736(18)31940-8:747-57

10 Petrovski S, Aggarwal V, Giordano JL, Stosic M, Wou K, Bier L, Spiegel E, Brennan K, Stong N, Jobanputra V, Ren Z, Zhu X, Mebane C, Nahum O, Wang Q, Kamalakaran S, Malone C, Anyane-Yeboa K, Miller R, Levy B, Goldstein DB, Wapner RJ. Whole-exome sequencing in the evaluation of fetal structural anomalies: a prospective cohort study. Lancet 2019;393:S0140-6736(18)32042-7:758-67.

11 Sparks TN, Lianoglou BR, Adami RR, Pluym ID, Holliman K, Duffy J, Downum SL, Patel S, Faubel A, Boe NM, Field NT, Murphy A, Laurent LC, Jolley J, Uy C, Slavotinek AM, Devine P, Hodoglugil U, Van Ziffle J, Sanders SJ, MacKenzie TC, Norton ME. Exome sequencing for prenatal diagnosis in nonimmune hydrops fetalis. N Engl J Med 2020;383:1746-56.

12 Kohler JN, Turbitt E, Biesecker BB. Personal utility in genomic testing: a systematic literature review. Eur J Hum Genet 2017;25:662-8.

13 Lerner B, Marshall N, Oishi S, Lanto A, Lee M, Hamilton AB, Yano EM, Scheuner MT. The value of genetic testing: beyond clinical utility. Genet Med 2017;19:71:763-71.

14 Hull D, Davies G, Armour CM. Survey of the definition of fetal viability and the availability, indications, and decision making processes for Post-Viability termination of pregnancy for fetal abnormalities and health conditions in Canada. I Genet Couns 2016;25:543-51.

15 Richards S, Aziz N, Bale S, Bick D, Das S, Gastier-Foster J, Grody WW, Hegde M, Lyon E, Spector E, Voelkerding K, Rehm HL, ACMG Laboratory Quality Assurance Committee. Standards and guidelines for the interpretation of sequence variants: a joint consensus recommendation of the American College of medical genetics and genomics and the association for molecular pathology. Genet Med 2015;17:405-23.

16 Hume S, Nelson TN, Speevak M, McCready E, Agatep R, Feilotter H, Parboosingh J, Stavropoulos DJ, Taylor S, Stockley TL, Canadian College of Medical Geneticists (CCMG). Ccmg practice guideline: laboratory guidelines for next-generation sequencing. J Med Genet 2019;56:792-800.

17 Boycott K, Hartley T, Adam S, Bernier F, Chong K, Fernandez BA, Friedman JM, Geraghty MT, Hume S, Knoppers BM, Laberge A-M, Majewski J, Mendoza-Londono R, Meyn MS, Michaud JL, Nelson TN, Richer J, Sadikovic B, Skidmore DL, Stockley T, Taylor S, van Karnebeek C, Zawati Ma'n H, Lauzon J, Armour CM, Canadian College of Medical Geneticists. The clinical application of genome-wide sequencing for monogenic diseases in Canada: position statement of the Canadian College of medical geneticists. J Med Genet 2015;52:431-7. 